\title{
Adapting two American Decision Aids for Mild Traumatic Brain Injury to the Canadian Context Using the Nominal Group Technique
}

\author{
El Kebir Ghandour ${ }^{1,2}$ (1) - Lania Lelaidier Hould ${ }^{2} \cdot$ Félix-Antoine Fortier $^{2} \cdot$ Veronique Gélinas $^{2} \cdot$ Edward R. Melnick $^{3}$. \\ Erik P. Hess ${ }^{4}$. Eddy S. Lang ${ }^{5}$. Jocelyn Gravel ${ }^{6}$. Jeffrey J. Perry ${ }^{7,8} \cdot$ Natalie Le Sage $^{9}$. Catherine Truchon ${ }^{1}$. \\ Annie LeBlanc ${ }^{9} \cdot$ Alexander Sasha Dubrovsky $^{10}$. Marie-Pierre Gagnon ${ }^{11}$. Marie-Christine Ouellet ${ }^{12}$. \\ Isabelle Gagnon ${ }^{13}$. Suzanne McKenna ${ }^{14}$. France Légaré ${ }^{2}$. Louise Sauvé ${ }^{15} \cdot$ Tom H$_{\text {. van de Belt }}{ }^{16}$. \\ Éric Kavanagh ${ }^{17} \cdot$ Laurence Paquette $^{17} \cdot$ Anne-Catherine Verrette ${ }^{17} \cdot$ Patrick Plante $^{15} \cdot$ Richard J. Riopelle $^{18}$. \\ Patrick M. Archambault ${ }^{2,9}$ on behalf of the Canadian Critical Care Trials Group, the Canadian Traumatic Brain \\ Injury Research Consortium and the Network of Canadian Emergency Researchers
}

Accepted: 17 September 2020

(c) Springer Nature Switzerland AG 2020

\begin{abstract}
Background Decision aids are patient-focused tools that have the potential to reduce the overuse of head computed tomography $(\mathrm{CT})$ scans.

Objective The objective of this study was to create a consensus among Canadian mild traumatic brain injury and emergency medicine experts on modifications required to adapt two American decision aids about head CT use for adult and paediatric mild traumatic brain injury to the Canadian context.

Methods We invited 21 Canadian stakeholders and the two authors of the American decision aids to a Nominal Group Technique consensus meeting to generate suggestions for adapting the decision aids. This method encourages idea generation and sharing between team members. Each idea was discussed and then prioritised using a voting system. We collected data using videotaping, writing material and online collaborative writing tools. The modifications proposed were analysed using a qualitative thematic content analysis.

Results Twenty-one participants took part in the meeting, including researchers and clinician researchers $(n=9 ; 43 \%)$, patient partners $(n=3 ; 14 \%)$ and decision makers $(n=2 ; 10 \%)$. A total of 84 ideas were generated. Participants highlighted the need to clarify the purpose of the decision aids, the nature of the problem being addressed and the target population. The tools require sociocultural adaptations, better identification of their target population, better description of head CT utility,
\end{abstract}

Prior Presentation Preliminary results were presented as a poster presentation at the International Shared DecisionMaking Conference, Quebec City, QC, Canada, on 9 July, 2019. Preliminary results were also presented at the joint annual scientific meetings for the Canadian Traumatic Brain Injury Research Consortium and Canadian Critical Care Trials Group (24 January, 2019; Lake Louise, AB, Canada). These results were also presented at the annual scientific meeting for the Network of Canadian Emergency Researchers (28 March, 2019; MontTremblant, QC, Canada) and Preventing Overdiagnosis 2017 Conference (17-19 August, 2017; Quebec City, QC, Canada).

Digital features To view digital features for this article go to https://doi.org/10.6084/m9.figshare.12943274.

Electronic supplementary material The online version of this article (https://doi.org/10.1007/s40271-020-00459-y) contains supplementary material, which is available to authorized users.

Extended author information available on the last page of the article 
advantages and related risks, modification of the visual and written representation of the risk of brain injury and head CT use, and locally adapted, patient follow-up plans.

Conclusions This study based on a Nominal Group Technique identified several adaptations for two American decision aids about head CT use for mild traumatic brain injury to support their use in Canada's different healthcare, social, cultural and legal context. These adaptations concerned the target users of the decision aids, the information presented, and how the benefits and risks were communicated in the decision aids. Future steps include prototyping the two adapted decision aids, conducting formative evaluations with actual emergency department patients and clinicians, and measuring the impact of the adapted tools on CT scan use.

\section{Plain Language Summary}

A mild traumatic brain injury (also called concussion) can happen when the brain moves around in the skull after an impact to the head. A concussion is not a brain bleed and you cannot see a concussion. Concussions do not show up on a computed tomography (CT) scan. Brain bleeds do. Computed tomography scans are specialised X-ray machines that can detect serious brain injuries. Unfortunately, CT scan use also exposes patients to radiation and a future increased risk of cancer.

Shared decision making involves health professionals and patients making decisions together based on the best available evidence, health professionals' experience, and patients' values and preferences. Shared decision making improves appropriate diagnostic test use.

Two decision aids created in the USA are available to facilitate shared decision making regarding the use of head CT scans for patients with concussion. These decision aids are not fully adapted for use in Canada because the healthcare, social and legal context is different. Our study brought together patients and experts in the field of concussion and shared decision making to analyse these decision aids and propose adaptations that would increase their acceptance in Canadian emergency departments. We used a technique called the Nominal Group Technique to create a consensus about the most important changes to make to both original decision aids. The main adaptations needed for the Canadian context concerned avoiding information about cost and removing any information that does not change clinical management. This project will help us adapt two decision aids for clinical use in Canada and support appropriate CT scan use for patients with concussion.

\section{Key Points for Decision Makers}

Canadian patient partners, clinicians and decision makers want to avoid presenting information that will not change clinical management and potentially increase patient anxiety.

Decision aids need cultural adaptation before use in healthcare systems with different legal and socio-political contexts.

The Nominal Group Technique was helpful in creating a consensus among a group of patient partners, clinicians, decision makers and scientists about the most important modifications needed to adapt two decision aids for use in a different healthcare system.

\section{Introduction}

Mild traumatic brain injury (mTBI) is a frequent cause of consultation in emergency departments (EDs) [1,2]. Mild traumatic brain injury is defined as a mild insult to the head that can result in a brief period of altered level of consciousness with a Glasgow Coma Scale of 13-15 measured $30 \mathrm{~min}$ after the injury [3]. While evaluating patients with mTBI, emergency physicians need to rule out clinically important brain injury even though the risk is low [1]. Although head computed tomography (CT) scans can rule out brain injury, they expose patients to potentially harmful ionising radiation $[4,5]$ and increase the ED length of stay [6]. Experts recommend ordering head CT scans for adult and paediatric patients with mTBI only when indicated by a validated decision rule [7-10]. However, the implementation of these rules has led to mixed results from modest reductions $[11,12]$ to increased head CT scan ordering [10,13], thus potentially contributing to overdiagnosis [12, 14].

In this context, shared decision making (SDM) may reduce overuse of head CT scans and address overdiagnosis $[14,15]$. Shared decision making is the process in which a physician and a patient review the evidence about an intervention or diagnostic test, assess options including the option of not undergoing the intervention or test, identify patient values/preferences, deliberate and make an informed value congruent decision [16, 17].

Decision aids (DAs) are knowledge tools designed to facilitate SDM that have shown improved patient knowledge, satisfaction and engagement in EDs $[15,17,18]$. Two DAs have been developed in the USA to better inform patients 
about the appropriate use of head CT scans for mTBI: one for children (the Head CT Choice decision aid [19]) and one for adults (the Concussion or Brain Bleed decision aid [20]). The paediatric DA increased parent satisfaction and trust in emergency physicians $[18,21]$, and reduced overall 7-day imaging and length of stay in the ED [18]. The adult DA improved patient knowledge [22].

An important part of implementing new DAs in clinical practice is adaptation to the local context, although there remains a need for more research about how best to accomplish this [23, 24]. To the best of our knowledge, no head CT DAs have been adapted to the Canadian context. We hypothesised that the two American DAs would require social, cultural, and political adaptations to improve their acceptability and facilitate their adoption in Canadian EDs. These adaptations would be justified by significant differences between the two health systems that influence the practice and organisation of services in both countries.[25, 26]. Thus, we aimed to create a consensus among Canadian patient partners and emergency medicine and SDM experts about the adaptations needed to make the two American DAs acceptable for use in Canadian EDs.

\section{Methods}

\subsection{Design, Setting and Participants}

Our study combined the Nominal Group Technique (NGT) [27] to reach a consensus about the adaptations needed to be made to the DAs, and an inductive qualitative analysis [28]. We report our qualitative results following the Standards for Reporting Qualitative Research [29] (Electronic Supplementary Material [ESM]). After receiving ethics approval, we purposively invited a convenience sample of Canadian emergency medicine, SDM and mTBI experts, patients, decision makers and trainees to a 1-day meeting held in Quebec City on 25 May, 2017. Invited participants were chosen to represent a diverse group of patients, clinicians, decision makers and scientists from across Canada who had expertise with the management and delivery of care to adults and children with mTBI or for their expertise in SDM. Decision makers were invited from organisations in Canada that produce mTBI guidelines, promote the quality of life of patients and families/caregivers who live with mTBI and/or lead local trauma committees that oversee the delivery of care to patients with $\mathrm{mTBI}$. To ensure we included the paediatric perspective, we invited a caregiver with experience in paediatric $\mathrm{mTBI}$, three paediatric $\mathrm{mTBI}$ experts and a paediatric trauma committee decision maker. We also invited the two original DA authors to attend our meeting.
Based on patient-oriented research best practices [30], our research team included patients partners defined as patients or caregivers with a lived experience of mTBI or with experience in caring for patients with $\mathrm{mTBI}$ who partnered with our team at different stages of our project to make our project and its outputs more relevant for them. Thus, we designed this study with a patient partner, and invited three other patient partners to our meeting including a caregiver (a parent of a child with lived mTBI experience). We also involved these patient partners in implementing the consensus meeting results and co-authoring the current publication. Participants who could not attend in person were invited to join using video conferencing (GoToMeeting, Boston, MA, USA). The meeting was moderated by the principal investigator, who was also an emergency physician and clinician scientist in the field of mTBI and implementation science.

\subsection{Data Collection}

The NGT consists of a structured meeting where ideas related to predetermined questions are discussed in six steps (Fig. 1). This technique has been used in a wide range of research fields for problem identification, research question generation, development of solutions and priority setting $[31,32]$. Two weeks before the meeting, we shared three predetermined questions with participants through a collaborative wiki platform, a web-based space where the organisers could freely share information files with the participants (CTRC Canadian Head CT Patient Decision Aid Consensus Study_WikiTrauma). Question \#1: What changes should be made to the proposed American DA in order to adapt it for adult mTBI patients in the context of Canadian EDs? Question \#2: Which adult patient population should we target to use our DA? Question \#3: What changes should be made to the proposed American DA in order to adapt it for paediatric mTBI patients in the context of Canadian EDs?

During the meeting, participants discussed and reformulated Question \#2 to: "Should we engage adult patients with medium risk (i.e. with at least one moderate risk criteria from the Canadian Head CT rule) in SDM?" because participants felt there was uncertainty about the applicability of SDM for this population. We also collected sociodemographic data on sex, professions, trainee status, areas of expertise, province and country of residence, and organisation affiliation.

\subsection{Data Analysis}

The meeting was video recorded with the consent of participants. Then, a team member (FA) reviewed the recordings and produced verbatim quotes illustrating all the suggested 
Fig. 1 Nominal Group Technique (NGT) main steps used in our study ( adapted from Delbecq et al. [27])

\section{Step 1. Silent idea generation}

- Each question was displayed on a large screen announcing the first step of the NGT process. Participants spent up to 20 minutes to write their ideas for each predetermined question on a $3 \times 5$ inch index card provided by the research team.

\section{Step 2. Round-robin sharing of ideas}

- Each participant took turns to share one of their ideas with the group. Two research team members (MD and RF) recorded each new idea to a Google Docs

Spreadsheet (Mountain View, CA). Each participant shared new ideas until no new idea emerged. At this step, ideas were generated and recorded without discussion.

\section{Step 3. Discussion and clarification of ideas}

- The moderator then clarified and evaluated each idea through group discussion to exclude, modify or group similar ideas without trying to influence participants' answers. New ideas could also emerge at this phase. However, combining two or more distinct ideas into one was not allowed at this time. Discussions were planned for 45 minutes or less if data saturation occurred.

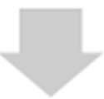

\section{Step 4. Ranking the suggestion}

- Each participant was then invited to individually prioritize each idea and rank their top five suggestions via a point-rating system, 5 points being given to the most important and 1 point given to the least important idea (first round of voting). Participants were asked to write ideas on another $3 \times 5$ inch index card with their attributed ranks in the bottom right corner of the card. While preserving anonymity of each voter, the facilitator collected the cards and recorded each ranking on the Google Docs Spreadsheet, which automatically computed total scores.

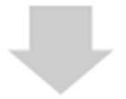

\section{Step 5. Presentation of the results}

- Using our online spreadsheet, we compiled the participants scores and ranks. Selected ideas were listed, beginning with the highest-ranked one. The table included a column indicating the score compilation for each idea along with the number of individuals who voted for each of them. We also pre-programmed bar charts that graphically supported the presentation of results in real time.

\section{Step 6. Discussion of the general classifications}

-The final ranking was compiled and presented to the participants for final discussion, clarification and modification. Both sessions' results were discussed at the same time at the end of the day followed by the meeting summary presentation. adaptations. In addition to counting votes for the ideas generated during the NGT, our qualitative thematic analysis developed a deeper understanding of the overall suggested changes to the original DAs. Another team member with qualitative methods expertise (MET) reviewed the verbatim transcripts and grouped similar ideological quotes into 
themes and counted their occurrence. These themes were reviewed and validated by four team members (PA, EG, LLH, VG).

\section{Results}

\subsection{Participants' Profile}

Out of 35 invited individuals, 21 participants took part in the meeting (Table 1), 18 in-person and three by video conferencing. Invited participants who could not attend
( $n=14)$ were similar to participants who attended, except for the proportion of decision makers (Table 1).

\subsection{Prioritisation of the Ideas Generated During the Nominal Group Technique}

Question \#1 generated a list of 46 ideas (ESM) about changes to make to the adult DA. The highest ranked ideas (Fig. 2) were: (1) changing the title to clarify the goal of the DA; (2) adapting the discharge plan to local contexts across Canada's different jurisdictions; and (3)
Table 1 Consensus meeting participants' characteristics

\begin{tabular}{|c|c|c|}
\hline & $\begin{array}{l}\text { Consensus meeting } \\
\text { participants }(n=21) \\
n(\%)\end{array}$ & $\begin{array}{l}\text { Invited participants who } \\
\text { did not attend }(n=14) \\
n(\%)\end{array}$ \\
\hline \multicolumn{3}{|l|}{ Sex } \\
\hline Male & $10(48)$ & $7(50)$ \\
\hline Female & $11(52)$ & $7(50)$ \\
\hline \multicolumn{3}{|l|}{ Primary area of expertise } \\
\hline Researchers & $13(62)$ & $9(69)$ \\
\hline EM experts/clinical experience & $5(24)$ & \\
\hline Shared decision-making experts & $2(9)$ & \\
\hline mTBI experts & $3(14)$ & \\
\hline Methodology experts & $3(14)$ & \\
\hline Decision maker & $1(5)$ & $2(15)$ \\
\hline Patients' representatives & $3(14)$ & $2(15)$ \\
\hline Quebec & $2(9)$ & \\
\hline Ontario (Brain Injury Canada, Ottawa) & $1(5)$ & \\
\hline Students (Université Laval, Québec, Canada) & $4(19)$ & \\
\hline Postdoctoral fellow & $1(5)$ & \\
\hline Emergency medicine resident & $1(5)$ & \\
\hline Epidemiology MSc Student & $1(5)$ & \\
\hline Medical student & $1(5)$ & \\
\hline \multicolumn{3}{|l|}{ Researchers' affiliations } \\
\hline Yale University (New Haven, USA) & $1(5)$ & \\
\hline Mayo Clinic (Rochester, USA) & $1(5)$ & \\
\hline Université Laval (Québec City, Québec, Canada) & $6(29)$ & \\
\hline Université de Montréal (Montreal, Canada) & $1(5)$ & \\
\hline McGill University (Montreal, Canada) & $1(5)$ & \\
\hline University of Ottawa (Ottawa, Canada) & $1(5)$ & \\
\hline University of Calgary (Calgary, Canada) & $1(5)$ & \\
\hline Dalhousie University (Halifax, Canada) & $1(5)$ & \\
\hline \multicolumn{3}{|l|}{ Country, Province } \\
\hline USA & $2(9)$ & \\
\hline Canada & $19(91)$ & \\
\hline Quebec & $15(72)$ & \\
\hline Ontario & $2(9)$ & \\
\hline Alberta & $1(5)$ & \\
\hline Nova Scotia & $1(5)$ & \\
\hline
\end{tabular}

$E M$ emergency medicine, $m T B I$ mild traumatic brain injury 
Fig. 2 Top ten ideas generated during the meeting for Question \#1
Number of voters

Total score after the votes

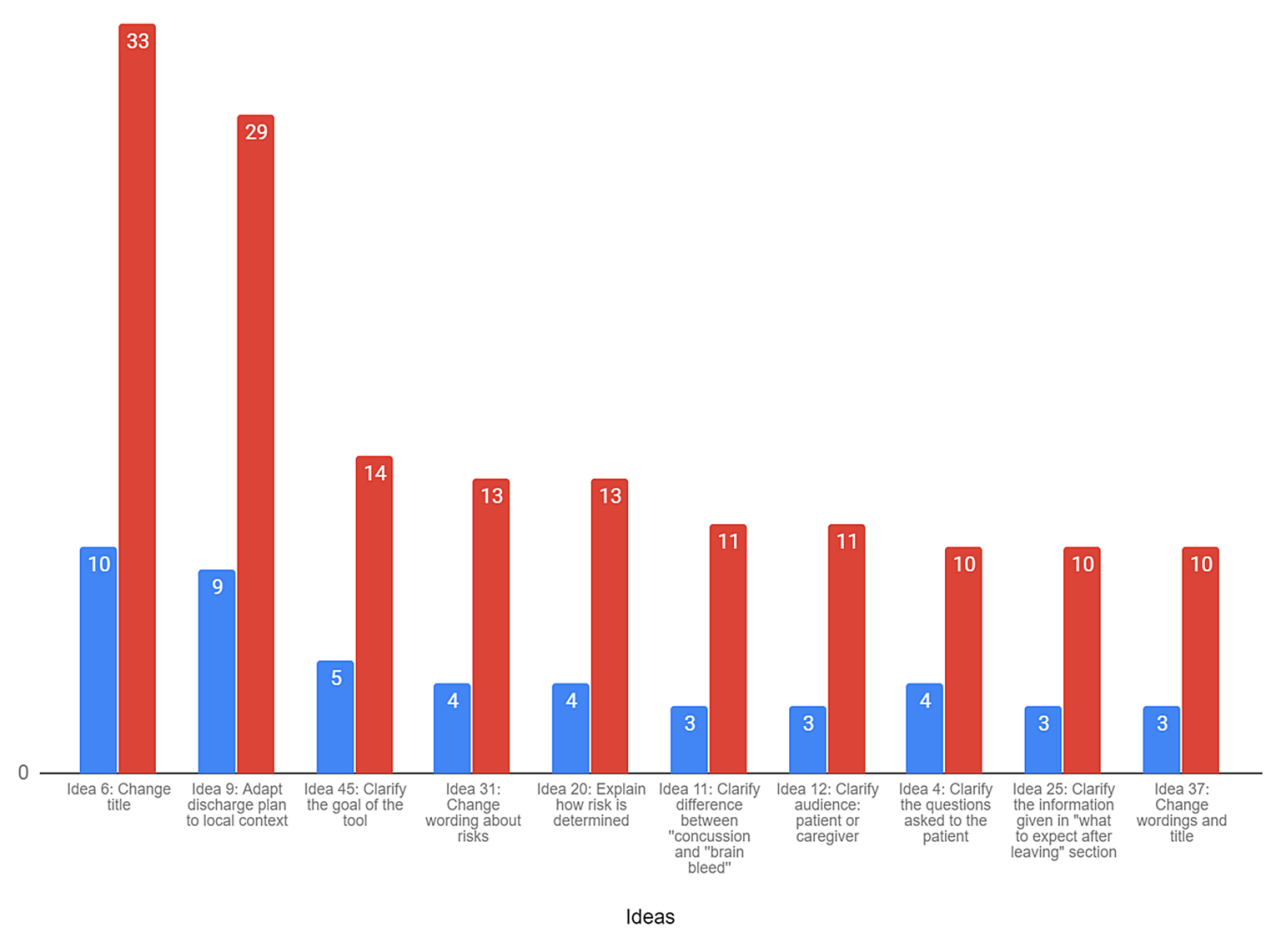

clarifying the role of the tool as a decision support tool vs a formal DA.

Prior to voting, participants discussed 15 different considerations about the use of SDM for medium-risk adult patients with mTBI to help clarify Question \#2 (ESM). All participants except one emergency physician voted in favour of employing SDM with medium-risk adult patients with mTBI (Fig. 3). This participant argued that SDM was not possible with these patients because they are often unable to make decisions because of their mTBI symptoms and are sometimes intoxicated.

Question \#3 generated 38 ideas (ESM) about changes to the paediatric DA. The highest ranked ideas (Fig. 4) were: (1) clarifying how TBI risk categories (high, medium, low) are presented to patients; (2) removing the issue of cost as a factor in decision making in Canada; and (3) explaining the difference between a concussion and a brain bleed.

\subsection{Thematic Analysis}

The 84 ideas generated during discussions for Questions $\# 1$ and 2, and the 15 ideas discussed for Question \#3 were analysed and classified into major themes and subthemes. Questions \#1 and 3 generated very similar themes and are presented together (Table 2), but Question \#2 generated its own set of themes (Table 3).

\subsubsection{Themes Discussed in Response to Questions \#1 and 3}

Three themes addressing different levels of adaptation were identified: need for clarification, risk communication and user friendliness.

\subsubsection{Need for Clarification}

Role of the Tool: Decision Aid or Decision Support The conceptual difference between DAs and decision support tools was not clear for participants. As participants compared the two DAs, it became clear that the adult tool was a decision support tool because it did not actually present formal options to patients like those found in the paediatric DA. Some changes were then suggested to make the tool's role clearer (Idea \#1.6; Table 2).

Definition of the Problem Being Addressed Additional clarification to the different terms used in the DAs was requested to ensure patients' understanding (Ideas \#1.11, 3.7 and 3.26; Table 2). Concussion, mild TBI and brain bleed are inconsistently used as synonyms, but the risk of complications associated with each diagnosis is not the same.

Targeted Population Participants mentioned that the tools' target population needed to be clearly identified to remove any ambiguity about their applicability (Ideas \#1.3 and 3.17; Table 2). For example, some participants wanted to know if the tools could be used with patients with minimal or trivial head injury. 
Fig. 3 Results of question "Should physicians engage in shared decision making with medium-risk adult patients?" (Number of voters $=14$, three experts and all trainees did not vote). $M D$ Physicians
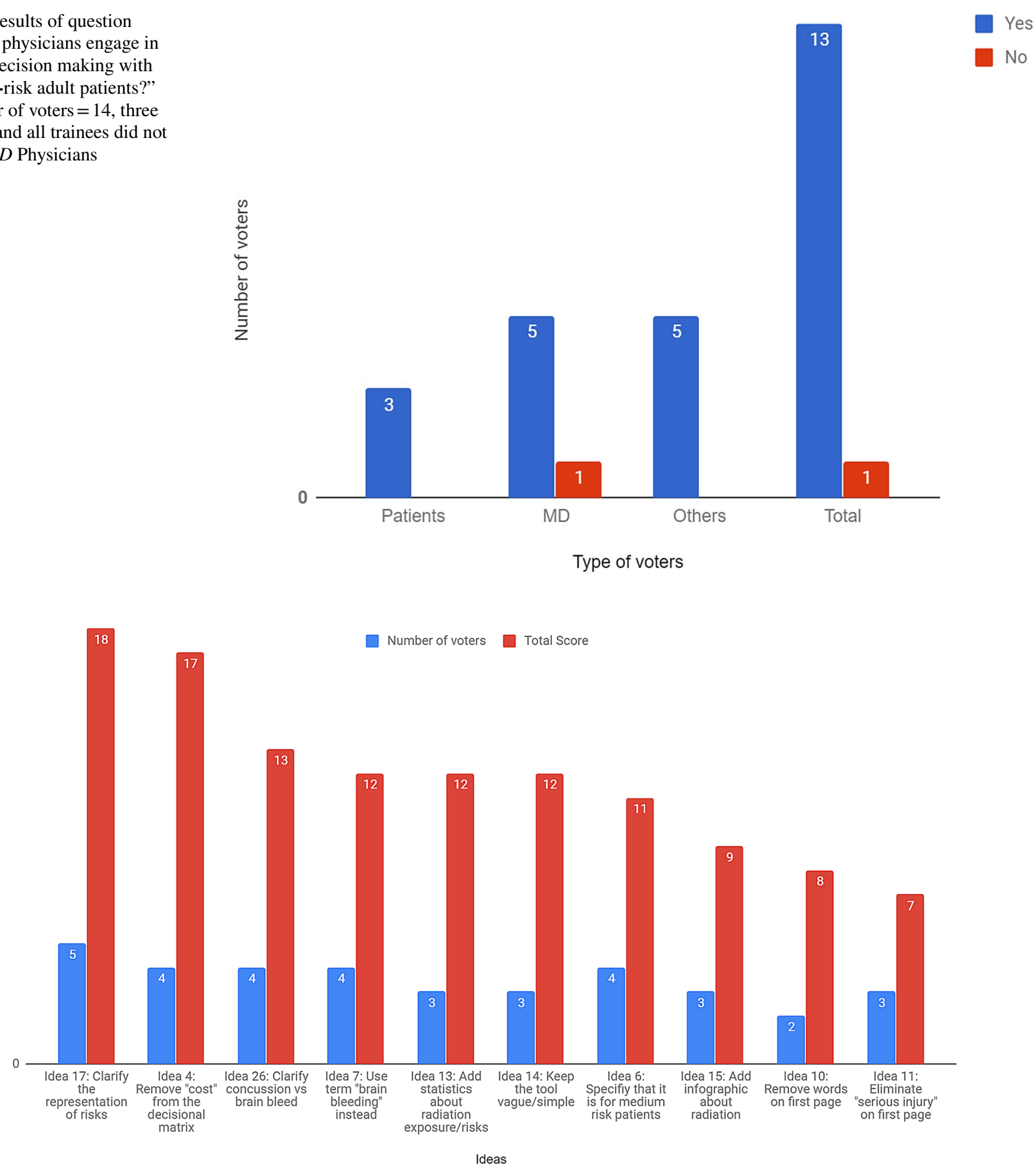

Fig. 4 Top ten ideas generated at the meeting for Question \#3

Sociocultural Adaptation Needed Participants suggested that sections not relevant to the Canadian context, such as the costs section, should be removed because Canadians do not pay directly for hospital-provided health services (Idea \#1.8 and 3.4; Table 2). Participants also suggested removing the representation of non-clinically significant brain injury (Idea \#1.5; Table 2). Canadian emergency physicians felt that presenting the risk of finding non-clinically significant brain injury to patients could potentially lead to overuse of head CT scanning and increase unnecessary anxiety in patients (Idea \#1.30; Table 2). In contrary, one American participant felt that this is an important concern for many emergency physicians in the USA who fear being sued even if they miss a non-clinically significant brain injury (Idea \#1.5; Table 2). Following this discussion, participants recommended changing the visual representation of the risk of 


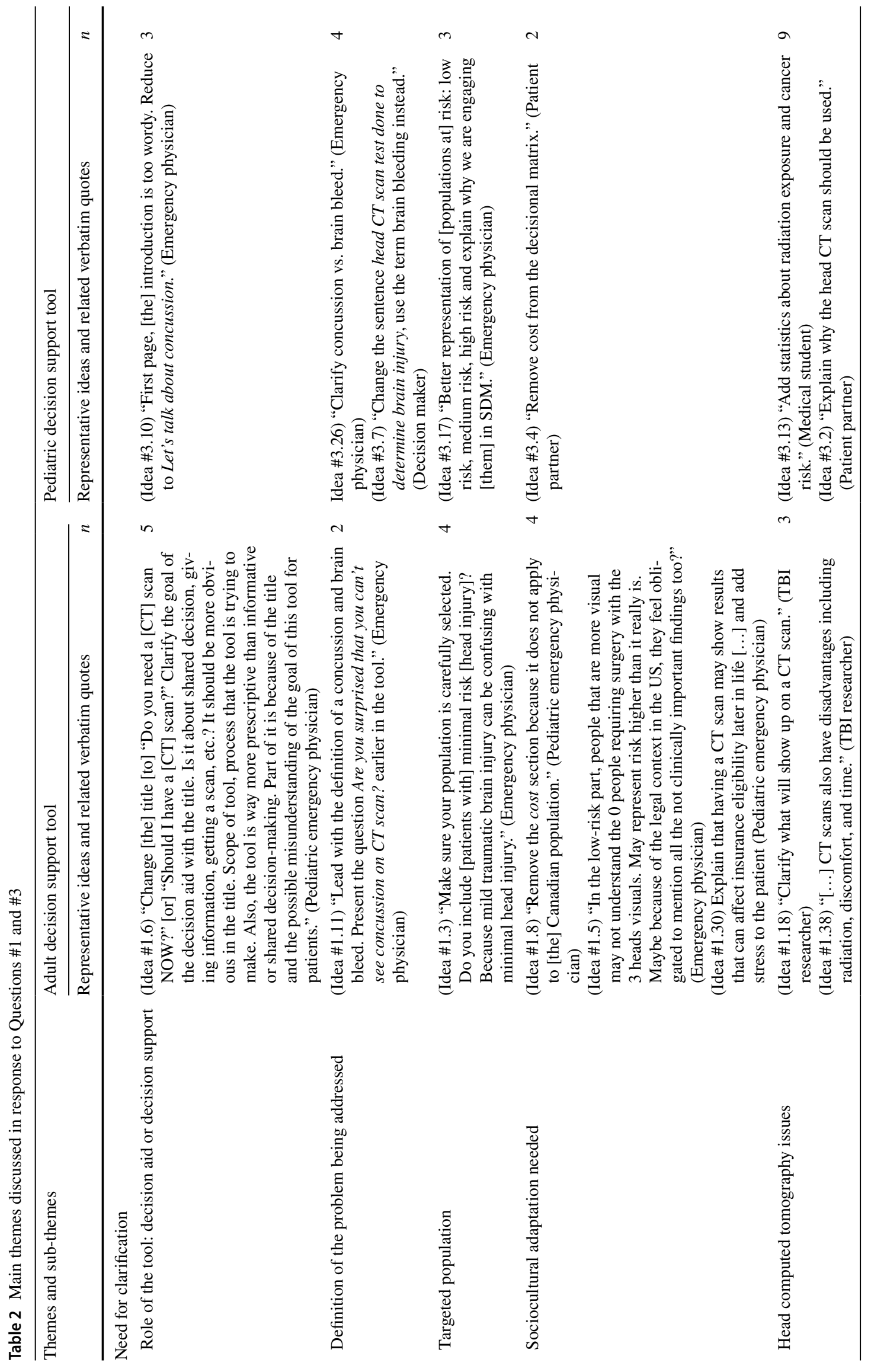




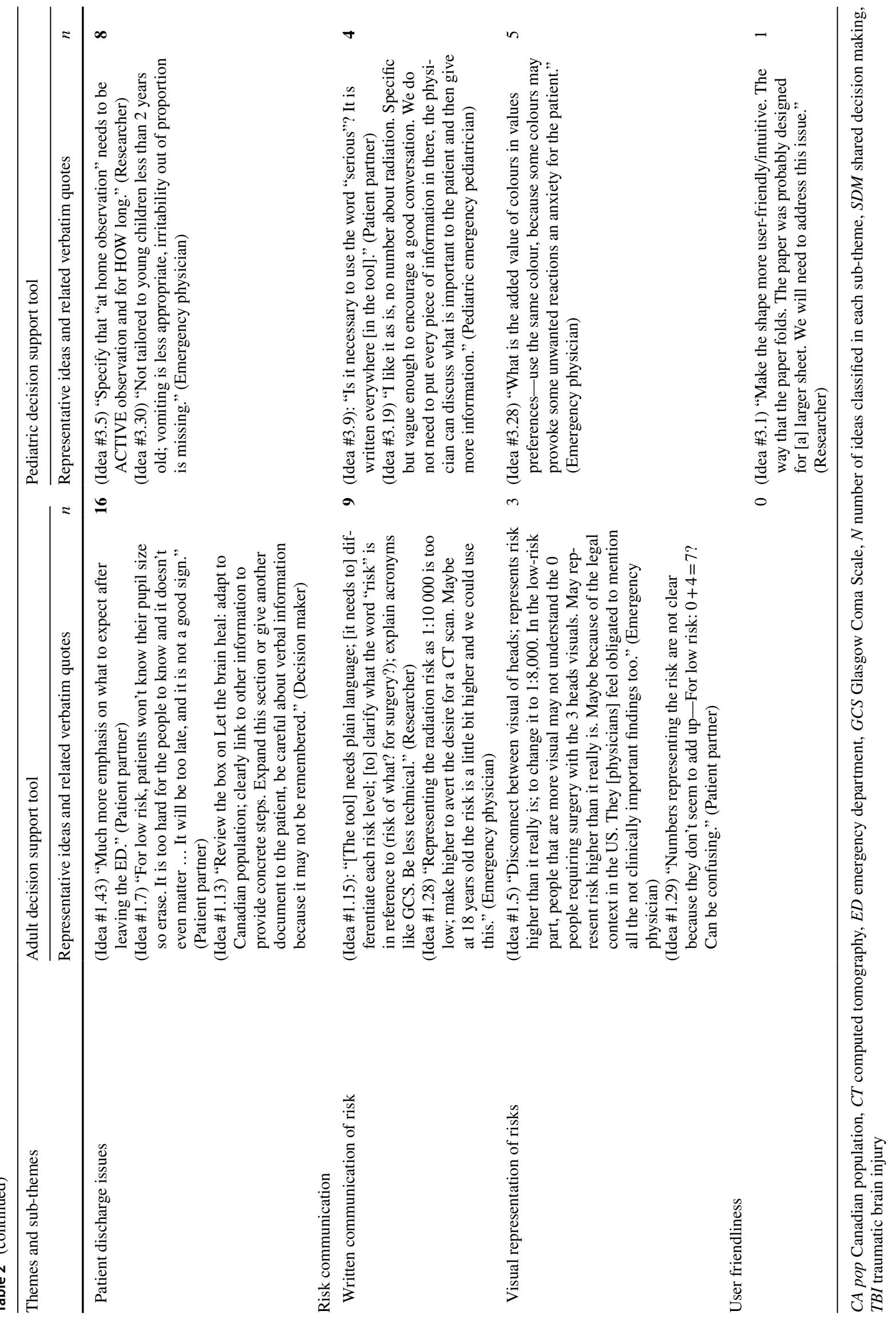


Table 3 Main themes discussed in response to Question \#2

\begin{tabular}{|c|c|c|}
\hline Themes and sub-themes & Representative ideas and related verbatim quotes & $n$ \\
\hline \multicolumn{3}{|l|}{ Clarification of the targeted population } \\
\hline \multicolumn{2}{|l|}{ SDM in medium-risk mTBI } & \\
\hline Shared decision is not possible for this population & $\begin{array}{l}\text { (Idea \#2.2) Not a high yield population in medium risk. Maybe there is not a } \\
\text { large number of cases at medium risk to begin with. Furthermore, they are } \\
\text { very symptomatic and often intoxicated. Is shared decision-making possible in } \\
\text { that situation? And if it is possible, would it really have an impact on the head } \\
\text { CT usage? (Emergency physician) }\end{array}$ & 3 \\
\hline Shared decision could be possible for this population & $\begin{array}{l}\text { (Idea \#2.3) Going after the medium risk group is the only way to reduce CT } \\
\text { scan overuse as they make up 50\% of TBI population. According to an emer- } \\
\text { gency physician, } 50 \% \text { of patients recruited in the Lancet study have medium or } \\
\text { high-risk criteria. They are all getting a CT scan because of the rule. Among } \\
\text { those, very little will have findings on the CT scan. So that's the category we } \\
\text { should focus on to reduce CT scans. (Emergency physician) } \\
\text { (Idea \#2.7) Patients would want to be involved in decision-making if medium } \\
\text { risk. (Two patient partners) }\end{array}$ & 4 \\
\hline Relevance of SDM for this population & $\begin{array}{l}\text { (Idea \#2.15) Need for a survey of patients and MDs' preferences about SDM in } \\
\text { middle risk }\end{array}$ & 3 \\
\hline Type of decision tool & $\begin{array}{l}\text { (Idea \#2.17) Tool should be more a decision support tool in the low-risk popula- } \\
\text { tion. If we make [this] a shared decision, patients may request a CT scan when } \\
\text { we know it is not necessary. By making [this] a shared decision, we could } \\
\text { even increase the CT [scan] rate in some situations.[...] The decision is sim- } \\
\text { ple; low risk equals no scan. However, in medium risk, it is possible to really } \\
\text { share the decision }\end{array}$ & 2 \\
\hline
\end{tabular}

$C T$ computed tomography, GCS Glasgow Coma Scale, MDs Physicians, $m T B I$ mild traumatic brain injury, SDM shared decision making, TBI traumatic brain injury

finding brain injury to only include the risk of finding clinically significant brain injury for the Canadian tools (Fig. 5).

Head Computed Tomography Issues Participants highlighted the importance of giving information about the utility, advantages and disadvantages of head CT scan use for mTBI management (Ideas \#1.38, 3.2 and 3.13; Table 2). Participants stressed the fact that patients need to understand that a concussion cannot be seen on a CT scan and that the main role of a head CT scan is to identify complications (e.g. a brain bleed) when clinically suspected. Radiation risk presented in the DAs was another major concern, especially in paediatric patients. Participants suggested that more information should be provided to help patients weigh the risk of undergoing or not a CT scan (Ideas \#1.38 and 3.13; Table 2).

Discharge Issues This sub-theme was the most discussed by patient partners and decision makers (Ideas \#1.7, 1.13 and 1.43; Table 2). They suggested improving the discharge instruction section to ensure that patients and parents feel confident in managing symptoms after discharge.

\subsubsection{Risk Communication}

Written Communication of Risk Participants discussed what risks need to be communicated to patients and par- ents (Ideas \#1.28 and 3.19; Table 2). The words used to describe risk and how risk is explained were highlighted as important matters to address. Additionally, trying to quantify and describe risk in detail could make our DAs too complex. Thus, participants highlighted the need to use plain language and simplify the description and quantification of risk (Ideas \#3.9 and \#3.19; Table 2).

Visual Representation of Risks Participants stated that the tool should provide precise information to patients while minimising unnecessary anxiety. Consistency between written and graphic information was not totally clear in the initial tool, thus changes were proposed to avoid ambiguity (Idea \#1.29; Table 2). The colours used in the figures were challenged because participants felt that some of them (e.g. the colour red) could be associated with negative emotional reactions (Idea \#1.28; Table 2).

3.3.1.3 User Friendliness Participants underscored the importance of making the paediatric tool more user friendly. During the meeting, participants had received an unfolded $8 \times 11$-inch format of the tool that was meant to be folded like a booklet. Once folded, the layout of the document was not intuitive, and the orientation of the pages was confusing. Participants suggested writing 


\section{Representation of low risk in original tool}

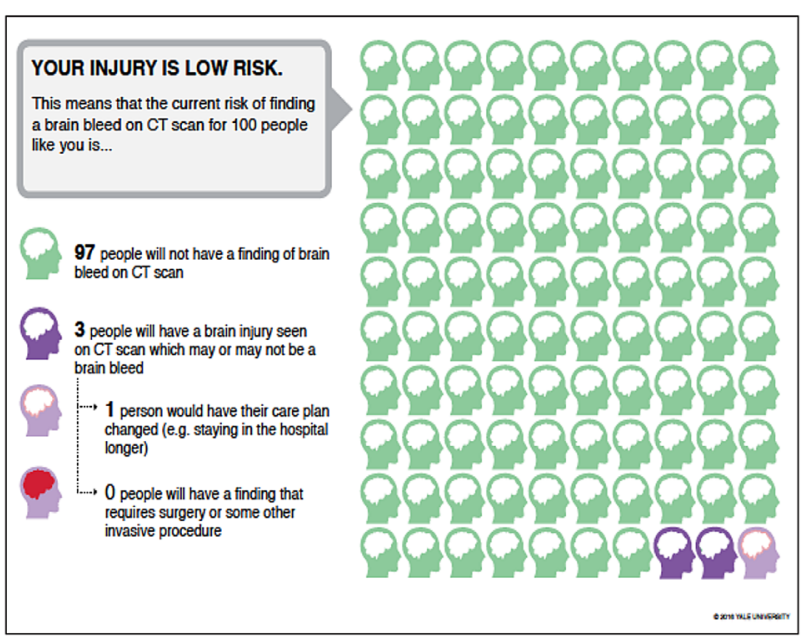

Representation of low risk in adapted tool

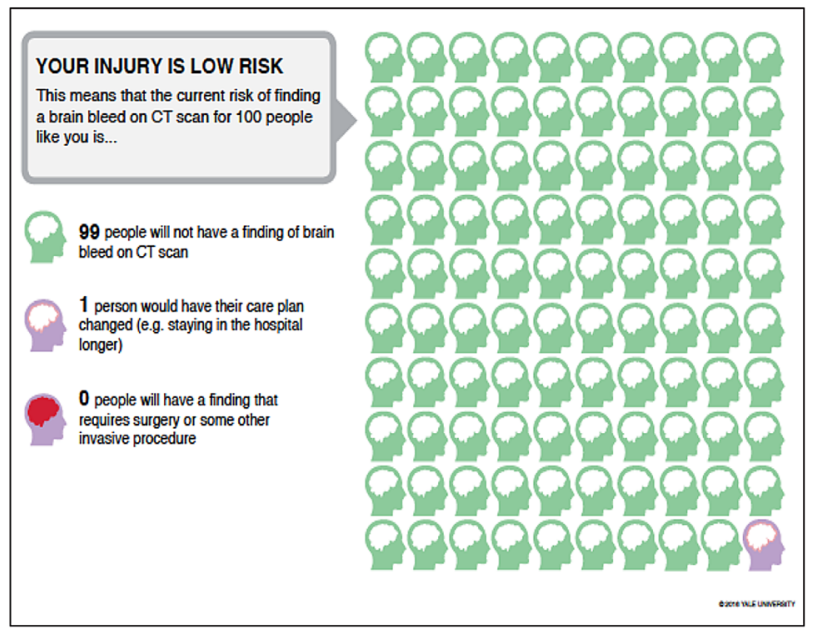

Fig. 5 Proposed graphic change to the adult decision support tool to avoid patient-clinician discussions about non-clinically significant findings on computed tomography (CT) scans

explicit printing specifications including double-sided printing (Idea \#3.1; Table 2).

\subsubsection{Themes Associated with Question \#2}

Question \#2 explored participants' willingness to use SDM with adults with medium-risk mTBI. Two themes were identified during these discussions: clarification of the targeted population and the possibility for SDM in patients with medium-risk mTBI.

3.3.2.1 Clarification of the Targeted Population Participants needed further clarification about medium-risk mTBI (i.e. patients with mTBI with Canadian Head CT Rule medium-risk criteria) vs moderate severity TBI (i.e. patients with TBI with a Glasgow Coma Scale < 13) (Table 3).

\subsubsection{Shared Decision Making in Medium-Risk Mild Trau- matic Brain Injury}

Possibility and Relevance for Shared Decision Making in Medium-Risk Mild Traumatic Brain Injury Only one participant felt that SDM was not possible with patients with medium-risk mTBI (Idea \#2.2; Table 3). Conversely, other participants recognised the potential utility of SDM with patients with medium-risk mTBI. Two patient partners expressed their desire to be involved in decision making for this risk category (Idea \#2.7; Table 3) and an emergency physician highlighted the fact that using SDM for this population could potentially reduce CT scan use (Idea \#2.3; Table 3). Given this debate, participants suggested surveying a larger sample of physicians and patients across Canada about their preferences on this topic (Idea \#2.15; Table 3).
Type of Decision Tool The type of tool most appropriate for patients with medium-risk mTBI remains unknown. Different perspectives were expressed concerning the usefulness of a DA, a decision support tool and a prognostic tool (Idea \#2.14 and 2.17; Table 3).

\section{Discussion}

We convened Canadian and American stakeholders including patients, clinicians, researchers and policy makers to recommend adaptations to two American DAs for use in Canada. Using a NGT, we collected a list of issues to address for the adaptation of the DAs to different sociocultural contexts. In particular, future adaptations for the Canadian context will need to clarify the role of the adult decision support tool and determine if a DA would be acceptable in medium-risk mTBI, ensure that patients understand the difference between terms used for brain injury, remove references to the cost of head CT scans, avoid presenting the risk of non-clinically significant brain injury and integrate discharge instructions adapted to local health services. The issues raised during this consensus meeting have led us to make the following observations.

First, the role of the tools needs to be clarified concerning whether they were DAs or decision support tools. Specific characteristics of each type of tool are defined in the literature but there is still an overlap between definitions [15, 33]. Decision aids actively engage patients in SDM while decision support tools inform patients without necessarily engaging them in decision making. The paediatric tool provides an explicit decision point for parents of children at medium risk of clinically important brain injury. In contrast, 
the adult tool is designed to support emergency physicians in the empathic sharing of information about head CT scan use in all mTBI risk categories (low, medium and high risk) without explicitly requiring decision making from the patient. The adult tool is more prescriptive in its recommendations to obtain a head CT scan for patients with high- and medium-risk mTBI [34]. This limits value-based decision making central to SDM. The participants suggested changes to the titles to avoid confusion about the tools.

Second, the choice of words to communicate risk needs clarification. The difference between concussion and brain bleed, as well as between the mTBI risk categories, may not be clear in the current DAs. Better communicating the criteria used to classify mTBI into low, medium or high risk could help parents understand the probability of finding a head trauma complication. This information is essential to make an informed decision about CT scan use. However, a debate exists about how much information should be given to patients when making evidence-based decisions [35]. Participants had divergent opinions about how to communicate the risk of brain bleeds vs the risk of radiation caused by the CT scan. Some participants were willing to de-emphasize the risk of a brain bleed and to emphasize the risk of radiation while others were not comfortable offering statistics on this topic. The increased lifetime risk of developing cancer after repeated exposure to radiation is an ongoing concern in the paediatric population [36]. Moreover, patients with low health literacy and numeracy might not understand the medical terms and statistics presented in the tools [37].

Third, the differences in legal and socio-political contexts between countries should be considered when adapting DAs. Currently, the American DAs may not be suitable for local language, legal context and the context of a publicly funded health system. Canadian emergency physicians and patient partners alike believed that findings that do not modify management should not be discussed with patients. Canadian participants thought that exposing patients to more uncertainty by presenting the risk of nonclinically significant brain injury when it does not change clinical practice could drive up patient anxiety and lead to overuse of head CT scanning by inflating the perceived risk of finding a brain injury. However, this could be relevant if DAs are used to reduce litigation risk in different legal contexts [38]. Furthermore, because most patients do not have to pay directly for public health services in Canada, participants suggested removing the CT scan cost section. Replacing "individual cost" with "cost for the society" was proposed as an alternative. These findings emphasise the need to consider the context when adapting tools, i.e. understand where, when and with whom these adapted DAs will be used [24].

Fourth, SDM might be used to engage patients with medium-risk mTBI more actively about head CT scan decision making. The current practice pattern based on the Canadian CT-Head rule is to order head CT scans for all patients with medium-risk mTBI [9]. Surprisingly, most participants including all the patient partners supported the use of SDM in this risk subgroup. This population represents a significant proportion of all mTBIs but among these, $4 \%$ have clinically significant injuries and none of them need neurosurgery [20,39]. Evidence from the USA supports the fact that patients prefer to be involved in decision making especially in the context of serious medical problems [40]. This supports the relevance of conducting a survey with a larger sample of emergency physicians and patients about their willingness to engage in SDM concerning head CT scan use in the medium-risk mTBI group. Wider interest in SDM for head CT scanning for patients with mTBI in the medium-risk category could support the relevance of developing a DA to address overdiagnosis in this population in the future.

Fifth, this study highlights the benefits of engaging patients when adapting DAs. During the consensus meeting, they helped integrate and understand patients' perspectives. Their lived experience of mTBI brought insights, concerns and priorities that were not considered before. Follow-up instructions were one of their concerns; as for many, the discharge information they received was not sufficient. Instructions need to be adapted to local healthcare service availability. These suggestions reflect the anxiety patients and parents face when it comes to being discharged back home. Finally, patient partners also helped us to use language more appropriate for patients. This inclusive approach to DA development and adaptation can potentially make these tools more user friendly and support the doctor-patient relationship [34].

\subsection{Limitations}

Our study has some limitations. First, we had to adapt the NGT to our larger group size and to the limited time available. We were not able to conduct two rounds of voting for each session, as normally recommended in a NGT. Second, participants invited to this meeting had an interest in using SDM with patients with mTBI. This likely biased the results of voting for question 2 . Third, in our analysis, we only used representative quotes of the ideas discussed and the number of times each idea was mentioned. However, to assure relevance, validity, and understanding, ideas, quotes and themes found through analyses were reviewed and validated by a multidisciplinary team of experts in mTBI and social science, as well as by some meeting participants. Fourth, we did not obtain feedback from patients and clinicians in realworld settings. This could have highlighted other changes to further adapt and improve our DAs for use with patients in Canadian EDs. In particular, only two paediatricians and 
one parent of a child with mTBI attended our meeting. We recognise that involving more paediatric experts and more parents could identify additional adaptations needed for the paediatric tool. To address this issue, our team is currently conducting user-centred design prototyping sessions with patients with mTBI, parents of children with mTBI, paediatricians, and more emergency physicians in two EDs including a specialised paediatric ED to validate and improve the proposed adaptations in both the adult and paediatric tools.

\section{Conclusions}

Our study based on an NGT identified several adaptations needed for two American DAs about the use of head CT scans for mTBI to support their use in Canada's different healthcare, social, cultural and legal context. These adaptations concerned the target users of the DAs, the information presented in the DAs, and the manner in which the benefits and risks were communicated. In particular, information that does not change clinical management or about costs needs to be adapted to the Canadian healthcare system's different organisational and clinical context, and to Canadian patients' values and preferences. Future steps include adapting the two DAs based on the ideas generated, conducting formative evaluations employing a user-centred design with actual ED patients and clinicians to ensure acceptability, and measuring the impact of the adapted tools on CT scan use.

Acknowledgements We thank Erik Hess and Edward Melnick for allowing us to adapt their original decision aids. We thank all consensus meeting participants for their contributions to this project. We also thank Marie-Ève Trottier, Janet Curran, Holly Witteman and Carrie Anna McGinn for their scientific contributions to this project. We thank Noah Silverberg and Jeffrey Caron for peer reviewing our manuscript on behalf of the Canadian Traumatic Brain Injury Research Consortium. We thank Ian Ball for peer reviewing our manuscript on behalf of the Canadian Critical Care Trials Group. Finally, we also thank Marcel Émond, Chair of the Grants and Manuscript Committee for the Network of Canadian Emergency Researchers, François Lauzier, Chair of the Grants and Manuscript Committee for the Canadian Traumatic Brain Injury Research Consortium, and Bram Rochwerg, Co-chair of the Grants and Manuscript Committee for the Canadian Critical Care Trials Group for coordinating the peer review of our manuscripts in all three research networks.

\section{Declarations}

Funding This study was funded by a grant from the Canadian Traumatic Brain Injury Research Consortium (CTRC) [http://www.ctrcccrt.ca/], the Department of Family and Emergency Medicine at Université Laval, the Bourse postdoctorale TD - Fondation Hôtel-Dieu de Lévis and the Fonds de Recherche Québec - Santé (\#32978). These organisations did not have any role or influence in the research except the CTRC, which is a group of researchers that collaborate to promote and improve traumatic brain injury research. This group provided sci- entific input into the design and analysis of the results of this study in addition to critically reviewing the manuscript.

Conflicts of Interest/Competing Interests El Kebir Ghandour, Lania Lelaidier Hould, Félix-Antoine Fortier, Veronique Gélinas, Edward R. Melnick, Erik P. Hess, Eddy S. Lang, Jocelyn Gravel, Jeffrey J. Perry, Nathalie Le Sage, Catherine Truchon, Annie LeBlanc, Alexander Sasha Dubrovsky, Marie-Pierre Gagnon, Marie-Christine Ouellet, Isabelle Gagnon, Suzanne McKenna, France Légaré, Louise Sauvé, Tom H. van de Belt, Éric Kavanagh, Laurence Paquette, Anne-Catherine Verrette, Patrick Plante, Richard J. Riopelle and Patrick M. Archambault have no conflicts of interest that are directly relevant to the content of this article.

Ethics Approval This study received approval from the Research Ethics Committee at the Centre Intégré de Santé et Services Sociaux de Chaudière-Appalaches (ethical approval number \#MP-23-2017-393).

Consent to Participate We obtained an informed consent to participate in our study from all participants. The majority attended our consensus meeting in Quebec City. They signed a consent form including consent to being video recorded during the meeting.

Consent for Publication All authors approved the submission for publication of the current version of the manuscript.

Availability of Data and Material Authors consent that research data and material could be made available on request.

Code Availability Not applicable.

Authors' Contributions EG and PA conceived and designed the study, EG, LLH, FF and PA collected data; EG, LLH, FF, VG and MT analysed the data; EG, LLH, VG and PA drafted the manuscript; all authors contributed substantially to its revision and its final approval, and were in agreement before publishing; PA takes responsibility for the paper as a whole.

\section{References}

1. Kuppermann N. Pediatric head trauma: the evidence regarding indications for emergent neuroimaging. Pediatr Radiol. 2008;38(Suppl. 4):S670-S674674. https://doi.org/10.1007/ s00247-008-0996-5.

2. Rao DP, McFaull S, Thompson W, Jayaraman GC. Trends in self-reported traumatic brain injury among Canadians, 2005-2014: a repeated cross-sectional analysis. CMAJ Open. 2017;5(2):E301-E307307. https://doi.org/10.9778/cmajo.20160 115.

3. Head J. Definition of mild traumatic brain injury. J Head Trauma Rehabil. 1993;8(3):86-7.

4. Mathews JD, Forsythe AV, Brady Z, Butler MW, Goergen SK, Byrnes GB, et al. Cancer risk in 680,000 people exposed to computed tomography scans in childhood or adolescence: data linkage study of 11 million Australians. BMJ. 2013;346:f2360. https://doi.org/10.1136/bmj.f2360.

5. Smith-Bindman R, Lipson J, Marcus R, Kim K-P, Mahesh M, Gould R, et al. Radiation dose associated with common computed tomography examinations and the associated lifetime attributable risk of cancer. Arch Intern Med. 2009;169(22):2078-86. https:// doi.org/10.1001/archinternmed.2009.427. 
6. Parma C, Carney D, Grim R, Bell T, Shoff K, Ahuja V. Unnecessary head computed tomography scans: a level 1 trauma teaching experience. Am Surg. 2014;80(7):664-8.

7. Cheng AHY, Campbell S, Chartier LB, Goddard T, Magee K, McEwen J, et al. Choosing Wisely Canada ${ }^{\circledR}$ : five tests, procedures and treatments to question in emergency medicine. CJEM. 2017;19(S2):S9-17. https://doi.org/10.1017/cem.2017.1.

8. Kuppermann N, Holmes JF, Dayan PS, Hoyle JD Jr, Atabaki $\mathrm{SM}$, Holubkov R, et al. Identification of children at very low risk of clinically-important brain injuries after head trauma: a prospective cohort study. Lancet. 2009;374(9696):1160-70. https://doi.org/10.1016/S0140-6736(09)61558-0.

9. Stiell IG, Clement CM, Rowe BH, Schull MJ, Brison R, Cass D, et al. Comparison of the Canadian CT Head Rule and the New Orleans criteria in patients with minor head injury. JAMA. 2005;294(12):1511-8. https://doi.org/10.1001/jama.294.12.1511.

10. Melnick ER, Szlezak CM, Bentley SK, Dziura JD, Kotlyar S, Post LA. CT overuse for mild traumatic brain injury. Jt Comm J Qual Patient Saf. 2012;38(11):483-9.

11. Sharp AL, Huang BZ, Tang T, Shen E, Melnick ER, Venkatesh $A K$, et al. Implementation of the Canadian CT Head Rule and its association with use of computed tomography among patients with head injury. Ann Emerg Med. 2018;71(1):54-63. e2. https://doi.org/10.1016/j.annemergmed.2017.06.022.

12. Dayan PS, Ballard DW, Tham E, Hoffman JM, Swietlik M, Deakyne SJ, et al. Use of traumatic brain injury prediction rules with clinical decision support. Pediatrics. 2017;139(4):e20162709. https://doi.org/10.1542/peds.2016-2709.

13. Stiell IG, Clement CM, Grimshaw JM, Brison RJ, Rowe BH, Lee JS, et al. A prospective cluster-randomized trial to implement the Canadian CT Head Rule in emergency departments. CMAJ. 2010;182(14):1527-32. https://doi.org/10.1503/cmaj.091974.

14. Brodersen J, Schwartz LM, Heneghan C, O'Sullivan JW, Aronson JK, Woloshin S. Overdiagnosis: what it is and what it isn't. BMJ Evid Based Med. 2018;23(1):1-3. https://doi.org/10.1136/ ebmed-2017-110886.

15. Stacey D, Légaré F, Lewis K, Barry MJ, Bennett CL, Eden KB, et al. Decision aids for people facing health treatment or screening decisions. Cochrane Database Syst Rev. 2017;4:CD001431. https://doi.org/10.1002/14651858.CD001431.pub5.

16. Elwyn G, Frosch D, Thomson R, Joseph-Williams N, Lloyd A, Kinnersley P, et al. Shared decision making: a model for clinical practice. J Gen Intern Med. 2012;27(10):1361-7. https://doi. org/10.1007/s11606-012-2077-6.

17. Flynn D, Knoedler MA, Hess EP, Murad MH, Erwin PJ, Montori VM, et al. Engaging patients in health care decisions in the emergency department through shared decision-making: a systematic review. Acad Emerg Med. 2012;19(8):959-67. https ://doi.org/10.1111/j.1553-2712.2012.01414.x.

18. Hess EP, Homme JL, Kharbanda AB, Tzimenatos L, Louie JP, Cohen DM, et al. Effect of the head computed tomography choice decision aid in parents of children with minor head trauma: a cluster randomized trial. JAMA Netw Open. 2018;1(5):e182430. https://doi.org/10.1001/jamanetworkopen .2018 .2430 .

19. Hess EP, Wyatt KD, Kharbanda AB, Louie JP, Dayan PS, Tzimenatos $\mathrm{L}$, et al. Effectiveness of the head CT choice decision aid in parents of children with minor head trauma: study protocol for a multicenter randomized trial. Trials. 2014;15:253. https://doi. org/10.1186/1745-6215-15-253.

20. Melnick ER, Lopez K, Hess EP, Abujarad F, Brandt CA, Shiffman $\mathrm{RN}$, et al. Back to the bedside: developing a bedside aid for concussion and brain injury decisions in the emergency department. EGEMS (Wash DC). 2015;3(2):1136. https://doi.org/10.13063 /2327-9214.1136.
21. Skains RM, Kuppermann N, Homme JL, Kharbanda AB, Tzimenatos L, Louie JP, et al. What is the effect of a decision aid in potentially vulnerable parents? Insights from the head CT choice randomized trial. Health Expect. 2020;23(1):63-74. https://doi. org/10.1111/hex.12965.

22. Singh N, Hess E, Guo G, Sharp A, Huang B, Breslin M, et al. Tablet-based patient-centered decision support for minor head injury in the emergency department: pilot study. JMIR Mhealth Uhealth. 2017;5(9):e144. https://doi.org/10.2196/mhealth.8732.

23. Straus S, Tetroe J, Graham ID. Knowledge translation in health care: moving from evidence to practice. Chichester: Wiley; 2011.

24. Chenel V, Mortenson WB, Guay M, Jutai JW, Auger C. Cultural adaptation and validation of patient decision aids: a scoping review. Patient Prefer Adherence. 2018;12:321-32. https://doi. org/10.2147/PPA.S151833.

25. Ridic G, Gleason S, Ridic O. Comparisons of health care systems in the United States, Germany and Canada. Mater Sociomed. 2012;24(2):112-20. https://doi.org/10.5455/ msm.2012.24.112-120.

26. Chernomas R, Sepehri A. How to choose? A comparison of the U.S. and Canadian Health Care Systems. Abingdon-on-Thames: Taylor \& Francis; 2018.

27. Delbecq AL, Van de Ven AH. A group process model for problem identification and program planning. J Appl Behav Sci. 1971;7(4):466-92. https://doi.org/10.1177/002188637100700404.

28. Braun V, Clarke V. Using thematic analysis in psychology. Qual Res Psychol. 2006;3(2):77-101. https://doi.org/10.1191/14780 88706qp063oa.

29. O'Brien BC, Harris IB, Beckman TJ, Reed DA, Cook DA. Standards for reporting qualitative research. Acad Med. 2014;89(9):1245-51. https://doi.org/10.1097/acm.0000000000 000388 .

30. Archambault PM, McGavin C, Dainty KN, McLeod SL, Vaillancourt C, Lee JS, et al. Recommendations for patient engagement in patient-oriented emergency medicine research. CJEM. 2018;20(3):435-42. https://doi.org/10.1017/cem.2018.370.

31. Harvey N, Holmes CA. Nominal group technique: an effective method for obtaining group consensus. Int $\mathbf{J}$ Nurs Pract. 2012;18(2):188-94. https://doi.org/10.1111/j.1440172x.2012.02017.x.

32. Jones J, Hunter D. Qualitative research: consensus methods for medical and health services research. BMJ. 1995;311(7001):37680. https://doi.org/10.1136/bmj.311.7001.376.

33. Elwyn G, O'Connor AM, Bennett C, Newcombe RG, Politi M, Durand M-A, et al. Assessing the quality of decision support technologies using the International Patient Decision Aid Standards instrument (IPDASi). PLoS ONE. 2009;4(3):e4705. https://doi. org/10.1371/journal.pone.0004705.

34. Melnick ER, Hess EP, Guo G, Breslin M, Lopez K, Pavlo AJ, et al. Patient-centered decision support: formative usability evaluation of integrated clinical decision support with a patient decision aid for minor head injury in the emergency department. J Med Internet Res. 2017;19(5):e174. https://doi.org/10.2196/jmir.7846.

35. Naik G, Ahmed H, Edwards AGK. Communicating risk to patients and the public. Br J Gen Pract. 2012;62(597):213-6. https://doi. org/10.3399/bjgp12X636236.

36. Huang WY, Muo CH, Lin CY, Jen YM, Yang MH, Lin JC, et al. Paediatric head CT scan and subsequent risk of malignancy and benign brain tumour: a nation-wide population-based cohort study. Br J Cancer. 2014;110(9):2354-60. https://doi.org/10.1038/ bjc.2014.103.

37. Rootman I, Gordon-El-Bihbety D. A vision for a health literate Canada: report of the Expert Panel on Health Literacy. Ottawa, ON: Canadian Public Health Association. 2008.

38. Schoenfeld EM, Mader S, Houghton C, Wenger R, Probst MA, Schoenfeld DA, et al. The effect of shared decision making on 
patients' likelihood of filing a complaint or lawsuit: a simulation study. Ann Emerg Med. 2019;74(1):126-36. https://doi. org/10.1016/j.annemergmed.2018.11.017.

39. Stiell IG, Wells GA, Vandemheen K, Clement C, Lesiuk H, Laupacis A, et al. The Canadian CT Head Rule for patients with minor head injury. Lancet. 2001;357(9266):1391-6. https://doi. org/10.1016/s0140-6736(00)04561-x.
40. Schoenfeld EM, Kanzaria HK, Quigley DD, Marie PS, Nayyar N, Sabbagh $\mathrm{SH}$, et al. Patient preferences regarding shared decision making in the emergency department: findings from a multisite survey. Acad Emerg Med. 2018;25(10):1118-28. https://doi. org/10.1111/acem.13499.

\section{Affiliations}

El Kebir Ghandour ${ }^{1,2}$ (1) - Lania Lelaidier Hould ${ }^{2} \cdot$ Félix-Antoine Fortier $^{2} \cdot$ Veronique Gélinas $^{2} \cdot$ Edward R. Melnick $^{3}$. Erik P. Hess ${ }^{4}$. Eddy S. Lang ${ }^{5}$. Jocelyn Gravel ${ }^{6}$. Jeffrey J. Perry ${ }^{7,8} \cdot$ Natalie Le Sage $^{9} \cdot$ Catherine Truchon $^{1}$. Annie LeBlanc ${ }^{9}$. Alexander Sasha Dubrovsky ${ }^{10}$. Marie-Pierre Gagnon ${ }^{11}$ - Marie-Christine Ouellet ${ }^{12}$. Isabelle Gagnon $^{13}$. Suzanne McKenna ${ }^{14}$. France Légaré $^{9}$. Louise Sauvé ${ }^{15} \cdot$ Tom H. van de Belt $^{16}$. Éric Kavanagh ${ }^{17} \cdot$ Laurence Paquette $^{17} \cdot$ Anne-Catherine Verrette $^{17} \cdot$ Patrick Plante $^{15} \cdot$ Richard J. Riopelle $^{18}$. Patrick M. Archambault ${ }^{2,9}$ on behalf of the Canadian Critical Care Trials Group, the Canadian Traumatic Brain Injury Research Consortium and the Network of Canadian Emergency Researchers

El Kebir Ghandour

elkebir.ghandour@inesss.qc.ca

1 Institut National d'excellence en Santé et en Services Sociaux (INESSS), 2535, Boulevard Laurier, 5e étage, Québec, QC G1V 4M3, Canada

2 Centre Intégré de Santé et de Services Sociaux de Chaudière-Appalaches, Lévis, QC, Canada

3 School of Medicine, Yale University, New Haven, CT, USA

4 Department of Emergency Medicine, University of Alabama at Birmingham, Birmingham, AL, USA

5 Cumming School of Medicine, University of Calgary, Calgary, AL, Canada

6 Department of Pediatrics, Université de Montréal, Montreal, QC, Canada

7 Department of Emergency Medicine, University of Ottawa, Ottawa, ON, Canada

8 Clinical Epidemiology Program, Ottawa Hospital Research Institute, Ottawa, ON, Canada
9 Department of Family Medicine and Emergency Medicine, Université Laval, Québec, QC, Canada

10 Pediatrics and Pediatric Emergency Medicine, McGill University, Montreal, QC, Canada

11 School of Nursing, Université Laval, Québec, QC, Canada

12 School of Psychology, Université Laval, Québec, QC, Canada

13 School of Physical and Occupational Therapy, McGill University, Montreal, QC, Canada

14 Brain Injury Canada, Ottawa, ON, Canada

15 TELUQ, Québec, QC, Canada

16 Radboud University Medical Centre, Nijmegen, The Netherlands

17 School of Design, Université Laval, Québec, QC, Canada

18 Department of Neurology and Neurosurgery, McGill University, Montreal, QC, Canada 